\title{
It Takes More than the Past to Understand and Build the Archive
}

\section{Artist Contribution by Rosa Menkman}

Rosa Menkman's video contribution It Takes More than the Past to Understand and Build the Archive (2020), commissioned especially for this issue of Stedelijk Studies, tells the story of her renowned work A Vernacular of File Formats (2010). The work is an archive of a years-long research project into file formats, which are encoding systems that organize data according to a certain syntax. A Vernacular of File Formats brings together intentionally digitally broken or "glitched" images. It centers on one source image-a portrait of the artist herself-compressed with various file formats introduced with a similar error, revealing the unique aesthetic of how they organize data. Menkman's contribution sheds light on the decade since the work's creation. From its 2016 co-acquisition by the Museum of the Image, Breda, and the Stedelijk Museum Amsterdam to today, her video describes the historical, social, and chance contexts that originally produced this work, and how our relationship to our own images and data have evolved since-to deleterious effect.

\section{Biography}

Rosa Menkman is an artist whose work focuses on noise artifacts that result from accidents in both analogue and digital media, such as glitch, encoding, and feedback artifacts. From here, she sets out to facilitate an important insight into the otherwise obscure alchemy of standardization via resolutions. Through her research, which is both practice-based and theoretical, she uncovers resolutions that are anti-utopian, lost, unseen, or simply "too good to be implemented" in order to produce new ways to use and perceive through and with our technologies. 